\title{
Soil Analysis of Roads near Environment Soil for Lead Pollution Assessment, Study Case: Tehran- Karaj Highway
}

\author{
Mustafa Nur Istanbuly \\ Department of Sciences \& Environmental Engineering, Faculty of Natural Resources, University \\ of Tehran \\ Department of Renewable Natural Resources and Environment, Faculty of Agriculture \\ Engineering, University of Aleppo \\ istanbuly@ut.ac.ir \\ Bahman Jabbarian Amiri \\ Department of Sciences \& Environmental Engineering, Faculty of Natural Resources, University \\ of Tehran \\ Corresponding author \\ jabbarian@ut.ac.ir \\ Amir Hossein Hamidian \\ Department of Sciences \& Environmental Engineering, Faculty of Natural Resources, University \\ of Tehran \\ a.hamidian@ut.ac.ir
}

\begin{tabular}{|l|l|l}
\hline Submission date:- 21/5/2018 & Acceptance date:- 21/6/2018 & Publication date:- 5/9/2018
\end{tabular}

\begin{abstract}
There are different pollution sources, which affect soil including transportation, especially in highways. These effects can be assessed by Environmental Impact Assessment. In this study, seven sampling stations were selected in different distances $(0,10,20,50,100$ meter) along Tehran- Karaj highway. Samples were collected and their concentrations of $\mathrm{Pb}$ were measured using a FAAS after dry acid digestion. It was observed that the highway had a negative impact on the peripheral soil. These impacts were higher in Tehran with average lead concentrations, and declined by moving toward Karaj. Moreover, the average concentration of lead in soil significantly decreased by increasing distance from the roadside.

Highest lead concentration was in station (E) in the middle of the road with an average of 160.50 $\mathrm{mg} / \mathrm{kg}$. The highest average concentrations were observed in soils of the roadside with an average of 115.25 $\mathrm{mg} / \mathrm{kg}$. The highest concentration was also observed in station (B) and in the roadside, with a concentration of $302 \mathrm{mg} / \mathrm{kg}$, which was above the international allowable limits. Tehran- Karaj highway is in the list of the most polluted roads in the world and it must be concerned for its lead pollution, especially in the roadside. Essential steps for controlling the negative impacts must be taken including the expansion of green-space along the highway.
\end{abstract}

Keywords: Environmental impact assessment, Lead pollution, Tehran- Karaj highway.

Journal of University of Babylon for Engineering Sciences by University of Babylon is licensed under a Creative Commons Attribution 4.0 International License. 
Journal of University of Babylon for Engineering Sciences, Vol. (26), No. (8): 2018.

\section{تحليل تربة البيئة المحيطة بالطرق لتقييم مقدار التلوث بالرصاص (Pb)، منطقة الاراسة: الطريق السريع طهران - كرج \\ مصطفى نور استانبولي \\ قسم علوم و هنسة البيئة، كلية هندسة الدوارد الطبيعية، جامعة طهران \\ قسم الموارد الطبيعية المتجدة والبيئة، كلية المنسسة النراعية، جامعة حلب}

istanbuly@ut.ac.ir

بهمن جباريان اميري

قسم علوم وهنسة البيئة، كلية هندسة الموارد الطبيعية، جامعة طهران

الكاتب المسؤول

jabbarian@ut.ac.ir

(مير حسين حميديان

قسم علوم وهندسة البيئة، كلية هنسة الموارد الطبيعية، جامعة طهران

a.hamidian@ut.ac.ir

هناك العديد من مصادر التلوث التي تنؤثر على التربة، خاصةً في البيئة المحاذية للطرق السريعة. يمكن تفييم هذه الأثار

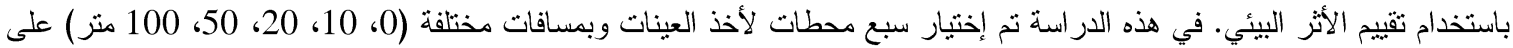
طول الطريق السريع طهران- كرج. تم قياس تركيز الرصاص للعينات المجموعة باستخدام جهاز الإمتصاص الذرب (FAAS) وذلاتك

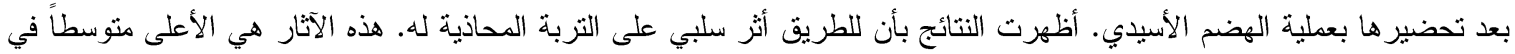
طهران؛ وتتتاقص بالتحرك بإتجاه كرج. بالإضافة إلى أن تركيز الرصاص ينتاقص بشكل معنوي بالإبتعاد عن حافة الطريق.

أعلى تركيز للرصاص تم تسجيله كمتوسط كان في المحطة (E) و الواقعة في الوسط على طول الطريق بمقار 160.50

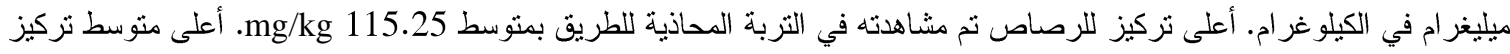

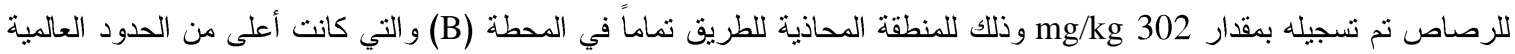
المسموح بها. حسب الدراسة يعتبر الطريق السريع طهران- كرج من أكثز الطرق الملوثة في العالم ويجبة التحذير من التلوث

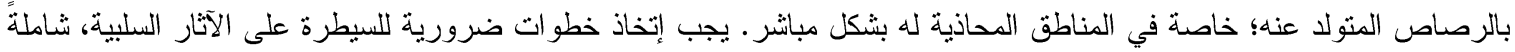
التوسع بالمساحلت الخضر اء على طول الطريق. الكلمات الداله: - تقييم الأثر البيئي، التلوث بالرصاص، طريق طهران- كرج. 
تعمل الأنثطة البشرية المتتوعة كالأثنطة الصناعية، الزراعية والإفادة من الوقود الإحفوري (نفط، بنزين، ديزل .... الخ)

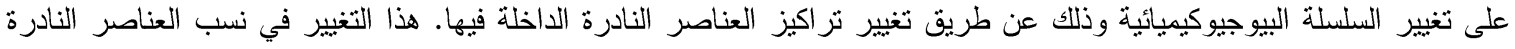

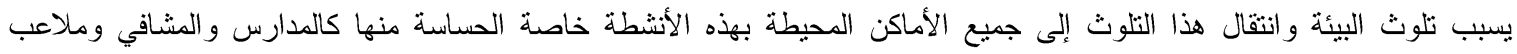

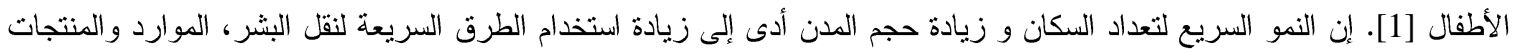

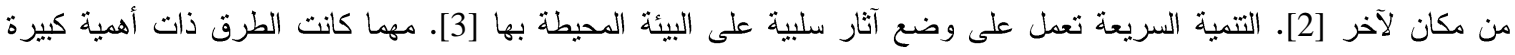

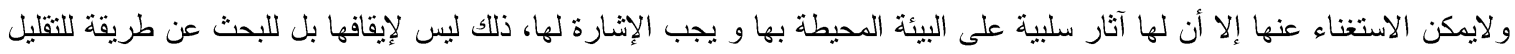

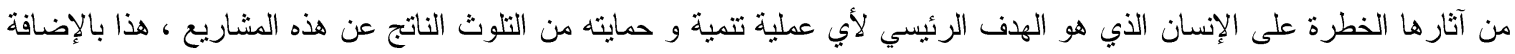

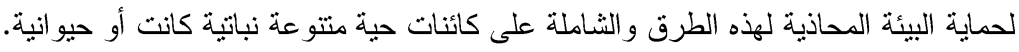

التربة هي إحدى الأوساط الرئيسة على الكرة الأرضية و هي كالجسم المنظّ و الواصل لعمليات دوران العناصر خاصة

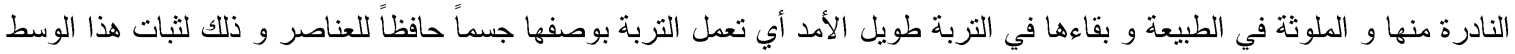

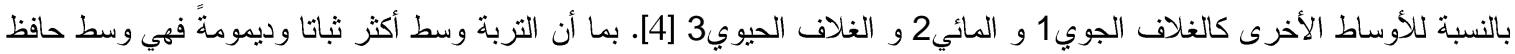

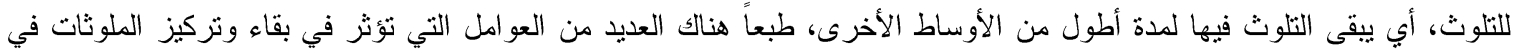

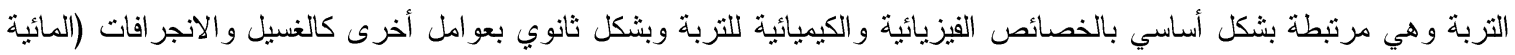

$$
\text { و الريحية) ..... الخ. }
$$

تتنقل العديد من الملوثات العضوية والمعدنية الناشئة عن الاستخدام المنكرر للطرق إلى البيئة المحيطة، ذلك إمّا عن طريق

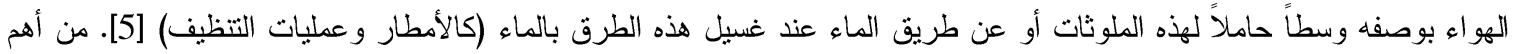

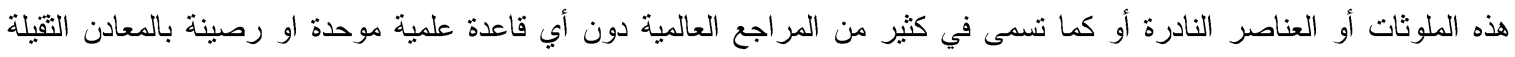

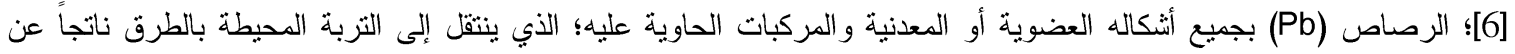

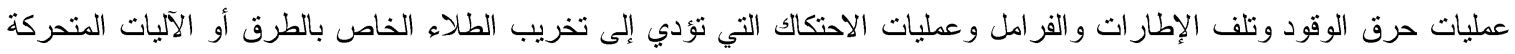

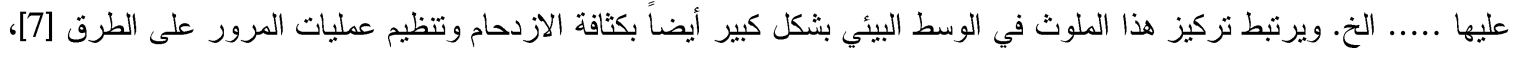

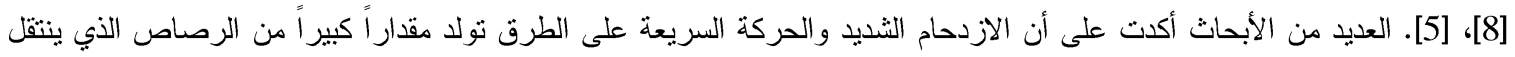

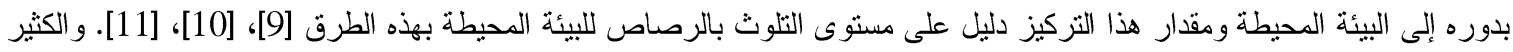

$$
\text { من ترب الأر اضي الزراعية المجاورة هي تحت معرض النلوث الناتج عن الطرقات [12]. }
$$

إن التتبؤ بهذه الملوثات ومر اقبتها يساعد متخذي القرار و المخططين على وضع خطط للحماية من الآثار السلبية لهذه الملوثات

(الرصاص منها) ودفع المجتمع أيضاً للذهاب في طريق التقليل من استخدام الموارد التي تعمل على توليد هذه الملوثات [1]

2. 2 الهذف من البحث

الهدف من الدر اسة الحالية هو تقييم مقدار التلوث بالرصاص التاتج عن عمليات احتراق الوقود خاصة للآليات المتحركة على كلى

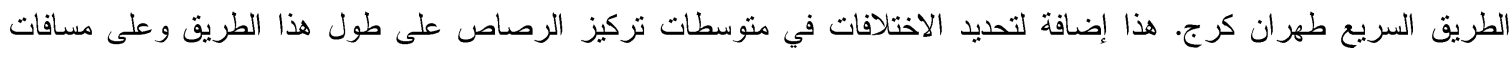

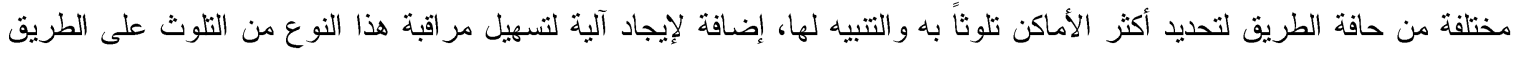

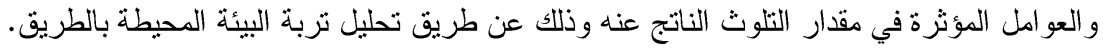

1 - Atmosphere

2 - Hydrosphere

3 - Biosphere 
Journal of University of Babylon for Engineering Sciences, Vol. (26), No. (8): 2018.

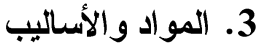

1.3. منطقة الدراسة

تقع منطقة الدراسة على الطريق الرئيسي والسريع الو اصل بين مدينة طهران ومدينة كرج في الجمهورية الإسلامية الإيرانية.

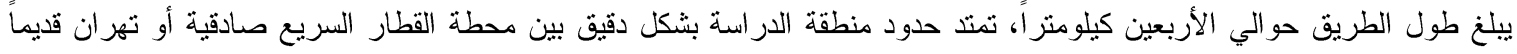

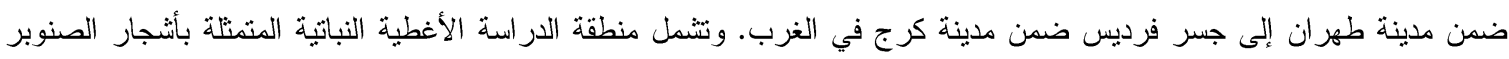
المزروعة على جو انب الطريق. الثكل (1).

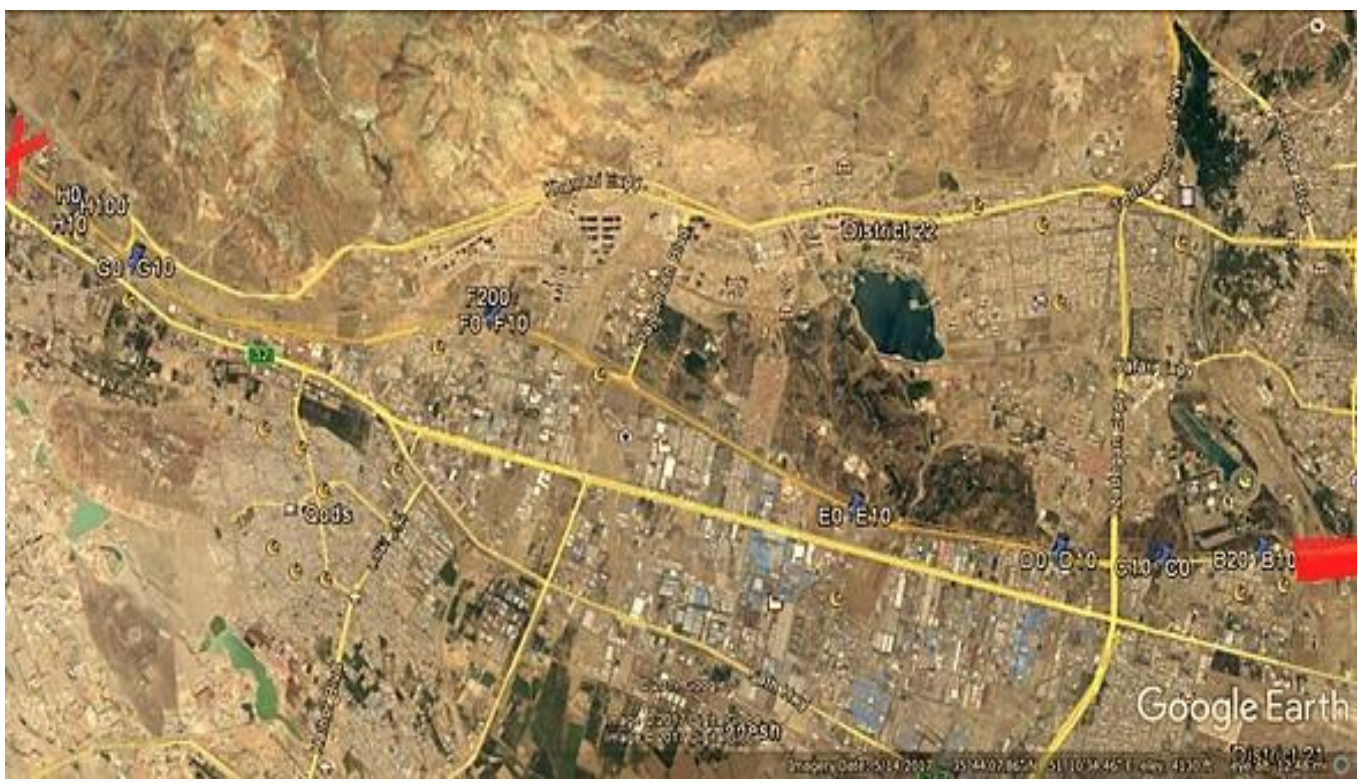

\section{شكل (1): محطات أخذ العينات على طريق طهران كرج (طهزان إلى يمين الصورة وكرج إلى يسار الصورة)}

شرح الثكل: يمتد طريق طهر ان كرج من يمين الثنكل إلى يساره بين العلامتين الموضحتين وتظهر محطات أخذ العينات والتي تم تحديدها بو اسطة

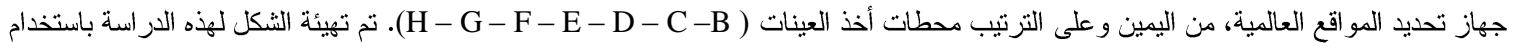

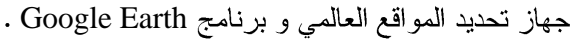

\section{3 طريقة الدراسة}

اختيرت سبع محطات لأخذ العينات على طول الطريق من مدينة طهران إلى مدينة كرج بحيث تكون بعيدة قدر الإمكان عن

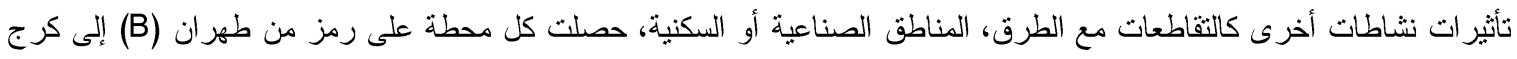

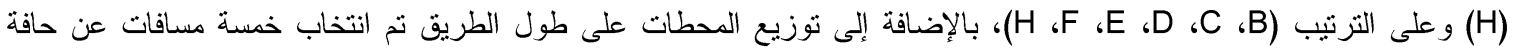
الطريق وكانت بالمثر (صفر، 10، 20، 50، 100) وذلك لدر اسة التغيرات في مقدار التلوث على طول الطريق وعلى مسافات مختلفة

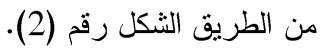

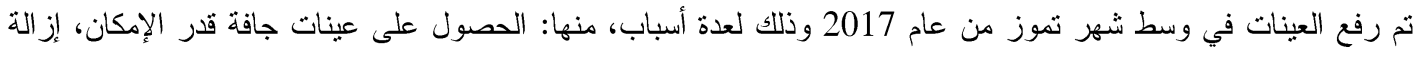

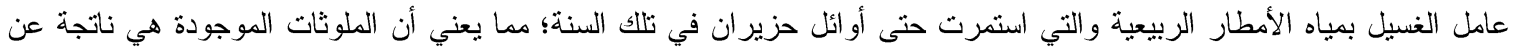

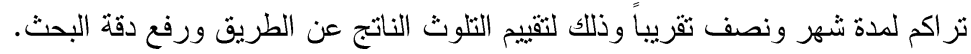




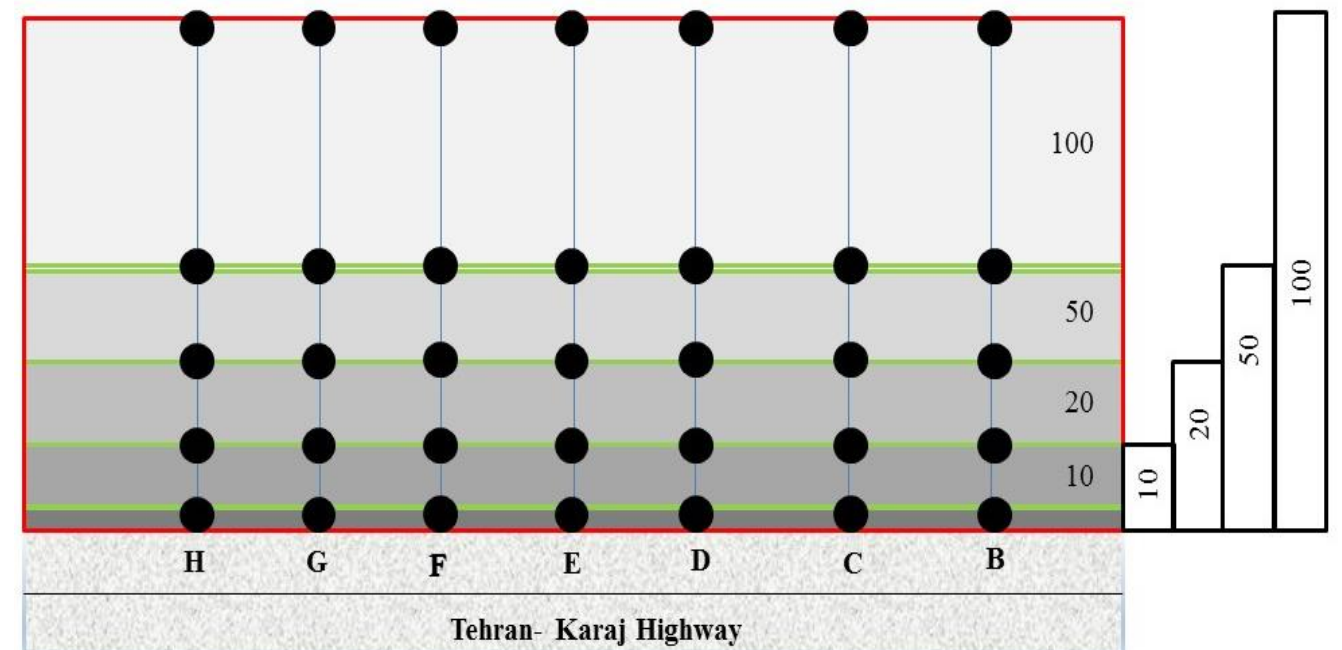

شكل رقم (2): طريقة توزيع مناطق أخذ العينات ضمن المحطات B - C - D - E - F - G - H (الدو ائر السوداء

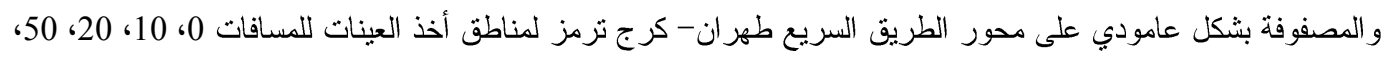
(ن) 100

كل عينة تربة هي عبارة عن خمس عينات جزئية (تحت عينة) بحيث أخذت بشكل دائرة ذات قطر نصف مثر و بعدد خمسة

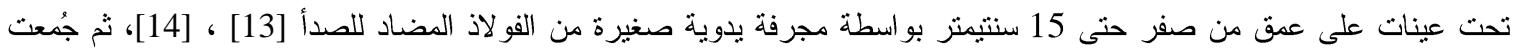

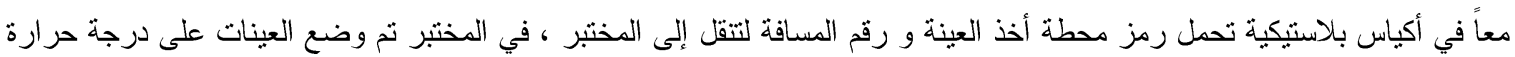

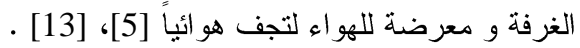

في المرحلة النالية والتي شملت تحضبر العينات للنحليل المخبري؛ في هذه المرحلة نم غربلة العينات بواسطة غربال ذي قياس

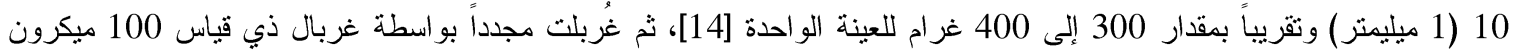

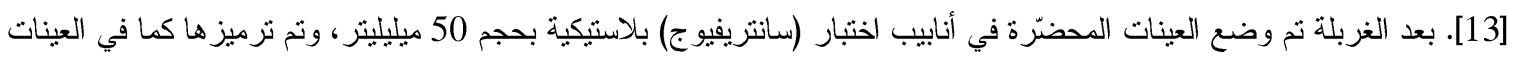
الحقلية (رمز المحطة ورقم المسافة) لمنع حصول أي تداخل بين العينات.

تم غسل جميع الأدوات المخبرية المستخدمة في تحضير العينات بالماء المقطر وثم وضعت لمدة 24 ساعة ضمن محلول حامضي بتركيز 4 \% مكون من ماء مقطر 96 جز هاء ماء مقر وأربعة أجزاء حمض النتريك (HNO3) وبعدها تم غسلها مجددا بالماء المقطر. في المرحلة الثالية استخدمت طريقة الهضم بواسطة الماء الملكي5 ، تكون فيها الأحماض الأسيدية المركزة بالنسبة 3:1 دائماً؛ أي جزء من حمض النتريك المركز (HNO3) لثلاثة أجز اء لحمض كلور الماء المركز (HCl)6؛ للارسة الحالية تم استخدام النسبة 3

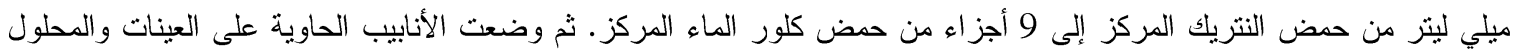

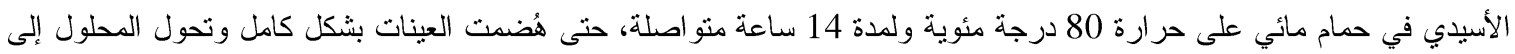

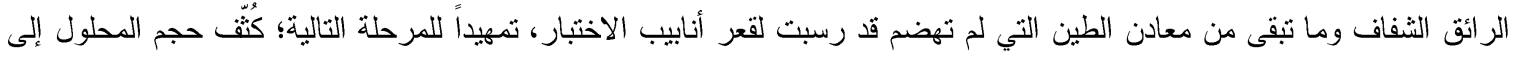

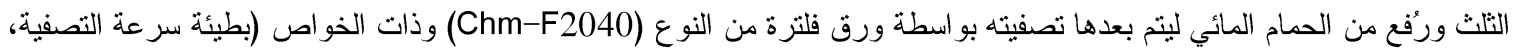

4 - Sup sample

5 - Aqua regia

6 - من و اجبي التحذير كون هذه العملية تحمل مخاطر كبيرة لأن هذه التركيبة تذيب أقوى المعادن ويجب أخذ دورات للتعامل مع هذه المواد واتخاذ إجرائات السلامة القصوى كون هذا الخليط يُنتج غاز ات بكميات كبيرة وقد يسبب انضغاط الأنابيب المغلقة و انفجرها مؤدياً إلى رشق الأسيد في موقع العمل 
فتحات ذات ثطر 7-9 ميكرومتر، محتوى رماد أقل من 007 \%) [15]. بعدها باستخدام بالونات زجاجية حجمية 50 ميلي ليتر نم

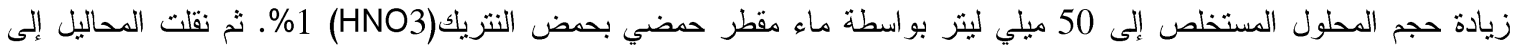
أو عية بلاستيكية نظيفة تماماً وذات حجم 50 ميلي ليتر، ثم وضعت في لئي البراد لحين إجراء تحليل تركيز معدن الرصاص فيهاء

تم قياس تركيز معدن الرصاص ضمن المحاليل باستخدام جهاز الامتصاص الذري من النوع ( OSK 6564 ATOMIC .(ABSORPTION SPECTROPHOTOMETER OGAWA SEIKI co. LTD AN 180

3.3. تم استخدام طرق الإحصاء الوصفي الثناملة على (المتوسطات، الوسيط، الوسط، الانحر اف المعياري واختبارات التوزيع

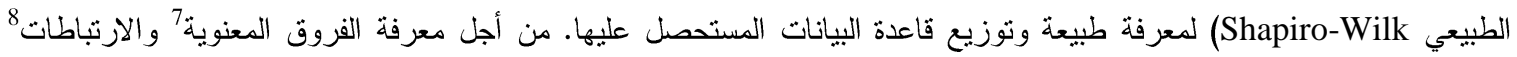
و الرو ابط والنمذجة تم استخدام اختبار (Kruskal-Wallis 1-way ANOVA) لكون الييانات لا تتنمي إلى التوزيع الطييعي

\section{4. تركيز الرصاص في ترب محطات أخذ العينات على طول الطريق من طهران إلى كرج}

من الجدول رقم (1) نجد بأن أعلى محطة تلوثاً بالرصاص المحطة الر ابعة (E) بمنوسط مساوي إلى 160.5 ميليغرام في

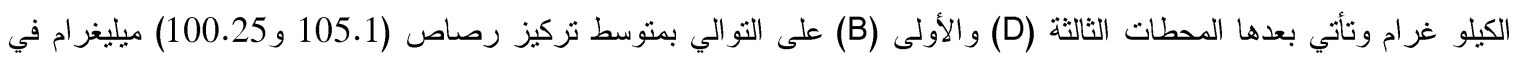

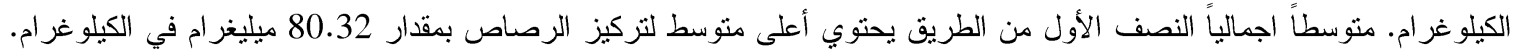

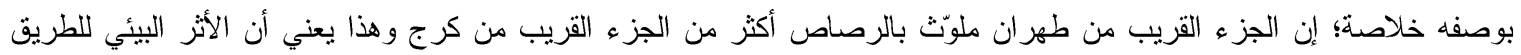

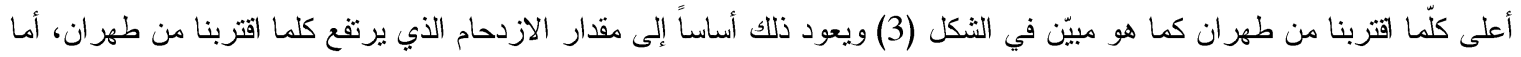

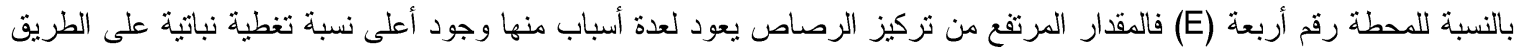

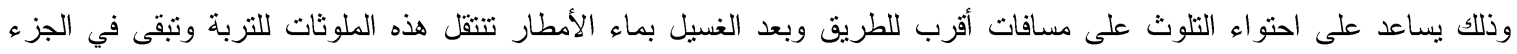

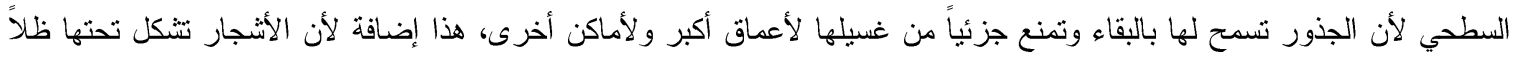

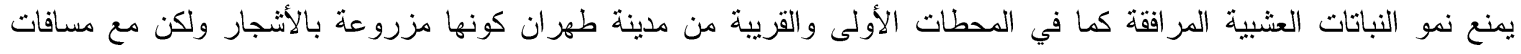
متباعدة مما سمح بتو اجد غطاء عثبي مر افق والذي يمنص الرصاص من التربة السطحية بو اسطة جذوره السطحية مما يقلل من تركيزه

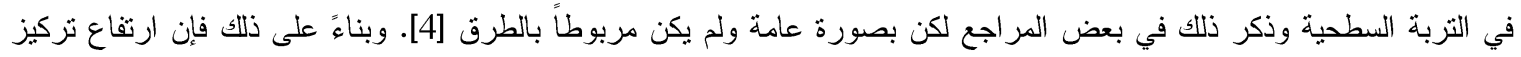
الرصاص في المحطة الر ابعة يعود أساساً لعدم تو اجد غطاء عثبي مر افق ولسبب آخر؛ هو أن هذه لهُ المنطقة تثكل نقطة اختناق مرورية

\begin{tabular}{|c|c|c|}
\hline \multicolumn{3}{|c|}{ جدول رقم (1):متوسط تركيز الرصاص في تربة محطات أخذ العينات } \\
\hline متوسط نركيز الرصاص (mglkg) & رمز المحطة & رقم المحطة \\
\hline 100.25 & B & 1 \\
\hline 35.60 & $\mathrm{C}$ & 2 \\
\hline 105.10 & $\mathrm{D}$ & 3 \\
\hline 160.50 & $\mathrm{E}$ & 4 \\
\hline 35.60 & $\mathrm{~F}$ & 5 \\
\hline 61.90 & G & 6 \\
\hline 46.50 & $\mathrm{H}$ & 7 \\
\hline
\end{tabular}
في بعض الأوقات.

7 - Difference's significant

8 - Correlations

9 - normal distribution, also known as the Gaussian or standard normal distribution 


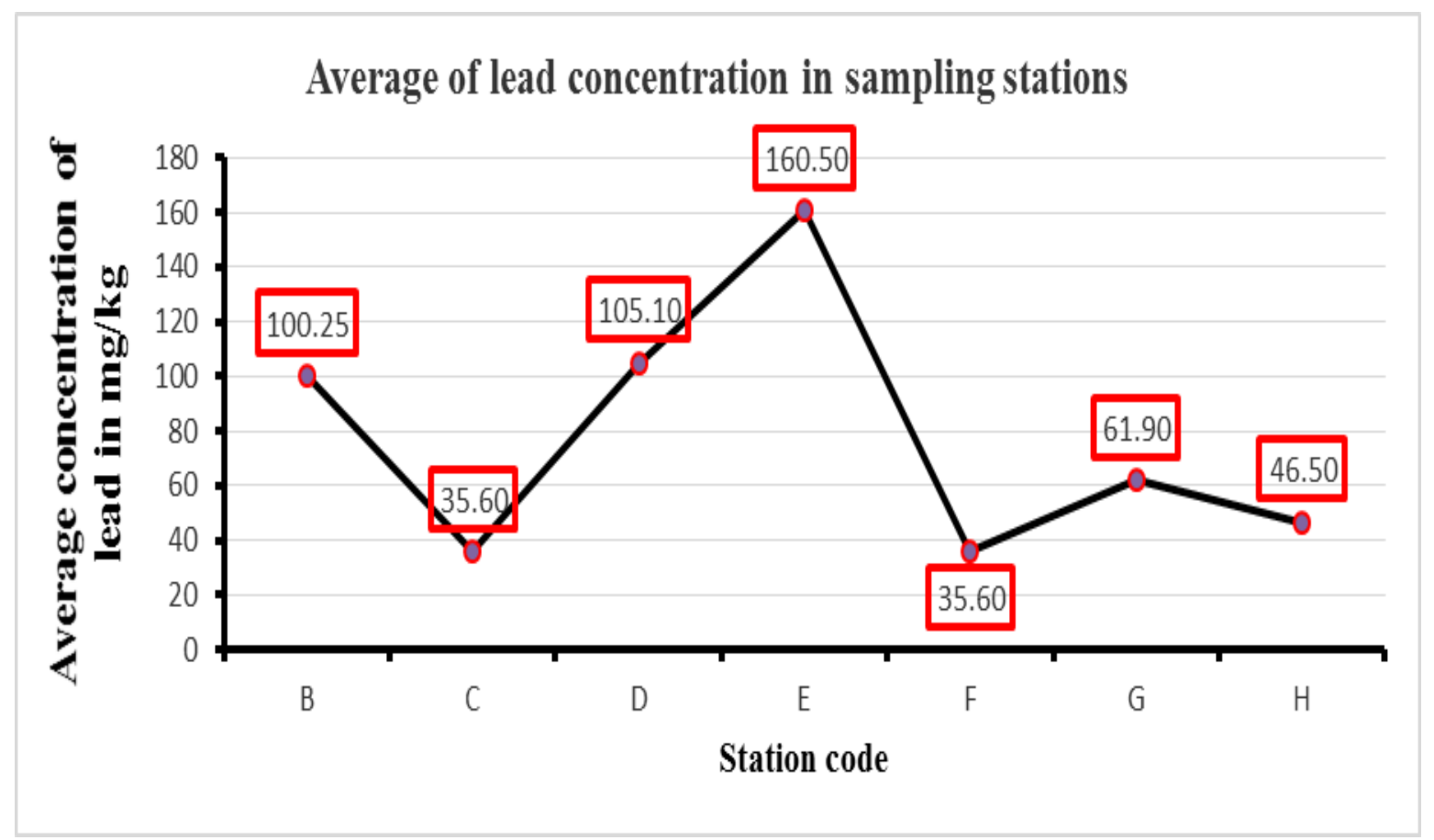

شكل (3): يظهز في الثكل متوسط تركيز الرصاص المقاس في محطات أخذ العينات (متوسط لجميع المسافات) من اليسار جانب طهران إلى اليمين بالقرب من كرج بالميليغرام في الكيلوغرام وذلك على في تربة محطات أخذ العينات، يظهر في الثكل أنه كلمّا ابتعدنا عن طهران قل التلوث.

2.4. ركيز الرصاص على مسافات مختلفة من حافة الطريق

مع الابتعاد عن حافة الطريق فإن تركيز الرصاص يقل تركيزاً بوصفه متوسطاً لجميع المحطات المدروسة، من 115.23 ميليغرام في الكيلوغرام وذلك للمسافة الأولى المواجهة للطريق مباشرة (صفر منر)؛ إلى المسافة 100 منز بمنوسط تركيز 52.92 ميليغر ام في الكيلوغر ام الجدول (2) و الثكل (4) وهذا يؤكد أن التلوث بالرصاص عائد للطريق ويقل مع الابتعاد عنه.

\begin{tabular}{|c|c|c|}
\hline ) وذلك ضمن مسافات مختلفة عن الطريق & ط تركيز الرصاص & 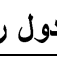 \\
\hline تركيز الرصاص (mg\Kg) & مقدار المسافة بالمتر & رقم \\
\hline 115.23 & 0 & 1 \\
\hline 61.23 & 10 & 2 \\
\hline 75.07 & 20 & 3 \\
\hline 78.43 & 50 & 4 \\
\hline 52.92 & 100 & 5 \\
\hline
\end{tabular}




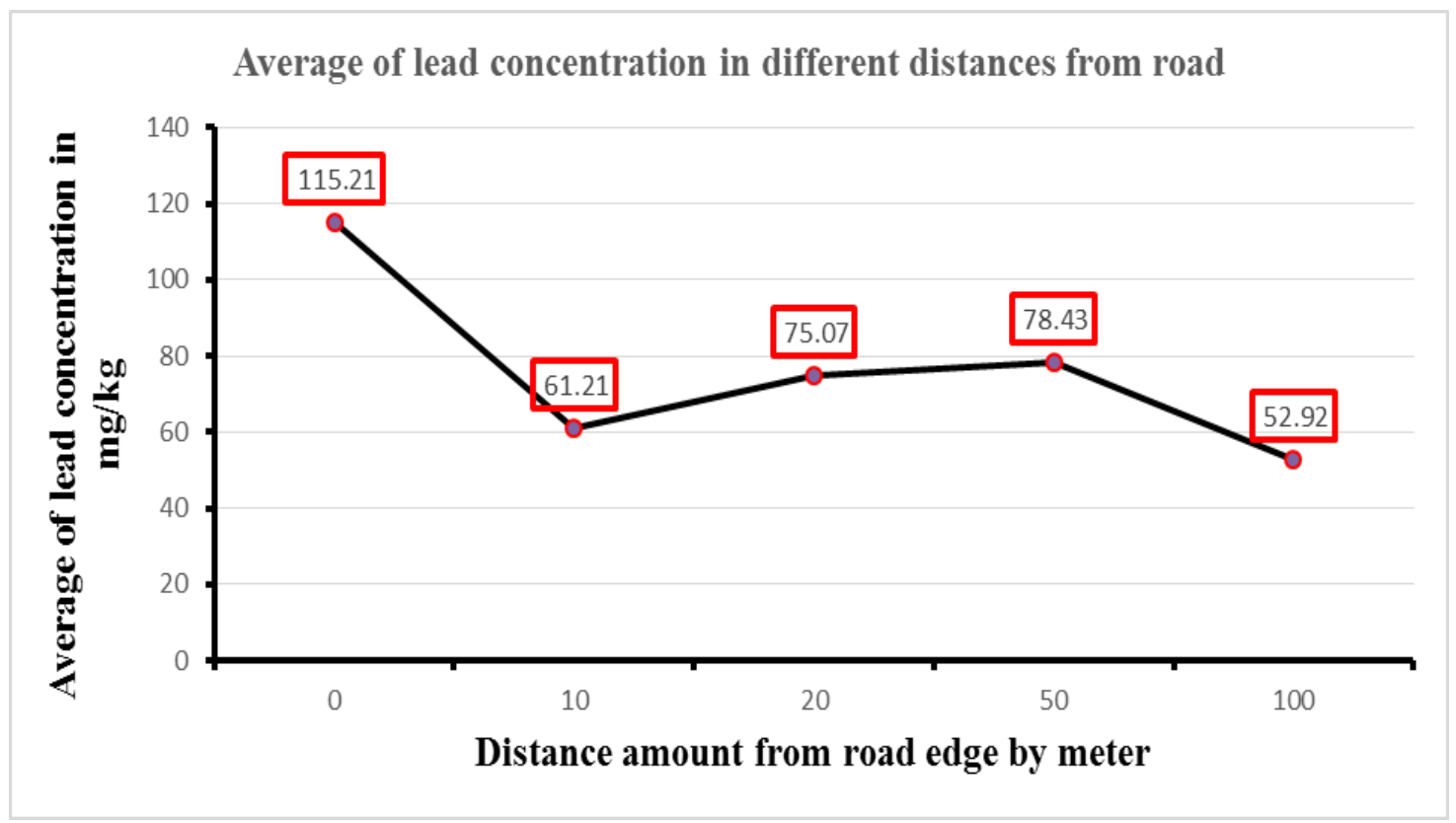

شكل رقم (4): متوسطات تركيز الرصاص بالميليغرام في الكيلوغرام والمسجلة من عينات التربة لمسافات مختلفة عن الطريق (0 - 10 -

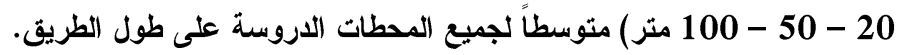

إن النتائج السابقة تحتاج للمقارنة مع مر اجع عالمية وذلك لمعرفة إن كان أثر الطريق ضمن الحدود المقبولة أو تجاوزها، وعند

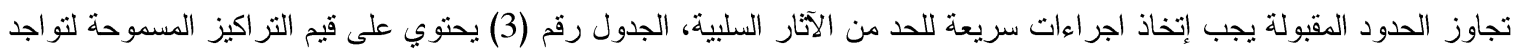
الرصاص في النباتات بناء على بعض الثقارير العالمية.

\begin{tabular}{|c|c|}
\hline \multicolumn{2}{|c|}{ جدول رقم (3)- التراكيز المسموحةً لتواجد الرصاص في النباتات بناء على بعض التثقارير العالمية } \\
\hline الحد الأعلى المنموح لثركيز الرصاص في ثربة (mg/kg) في الثباتات & المؤسنة صلاحبة النثريز \\
\hline 300 & {$[16] \mathrm{EU}^{10} 2005$} \\
\hline 350 & [17] SEPA $^{11} 2005$ \\
\hline 300 & [18] $\mathrm{CEPA}^{12} 1995$ \\
\hline 300 & [19] NZWWA ${ }^{13} 2010$ \\
\hline $2-300$ & [4] Kabata-Pendias 2011 \\
\hline
\end{tabular}


Journal of University of Babylon for Engineering Sciences, Vol. (26), No. (8): 2018.

و الجدول رقم (4) يحتوي على متوسطات نركيز الرصاص المسجلة في بعض الدراسات المتعلقة بالتلوث بالرصاص لتربة

الفاصلة الاولى المواجهة للطريق عبر العالم، حيث يحوي الجدول المذكور اسم الطريق المدروس ومنوسط تركيز الرصاص المسجل.

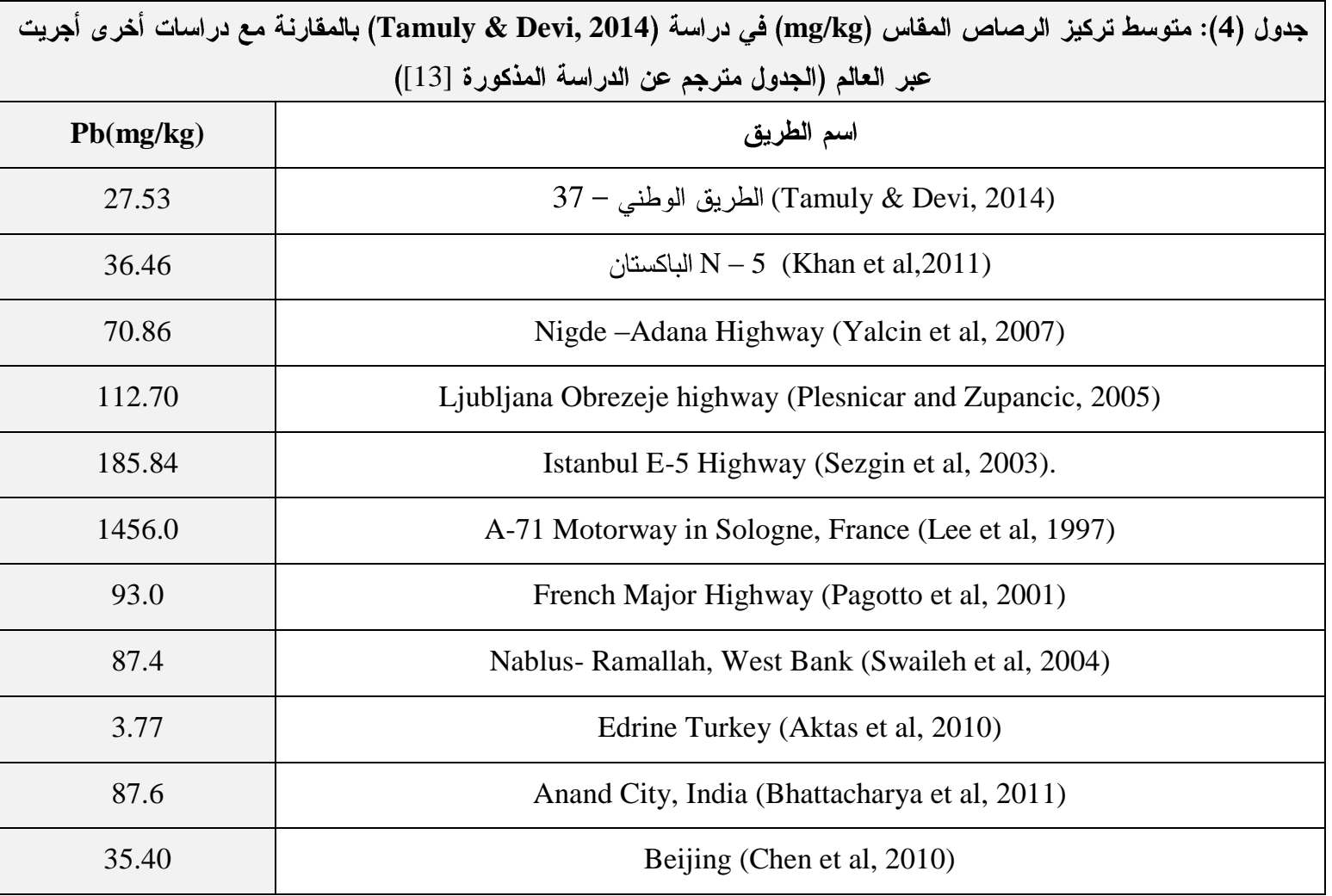

3.4. 3. 3. 3.

في البداية تم تحليل البيانات لمعرفة نوع التوزيع الإحصائي لها هل هو تابع للتوزيع الطبيعي او غير الطبيعي بإستخدام اختبار

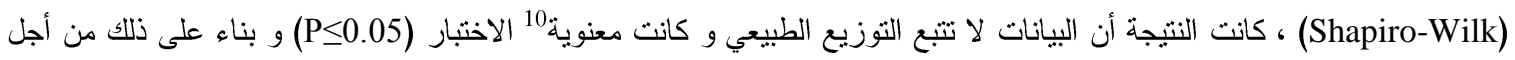
معرفة معنوية الفروقات بين المتوسطات تم استخدام اختبار (Kruskal-Walis) للبيانات غير البار امتزية (التي لا تتبع التوزيع الطييعي)

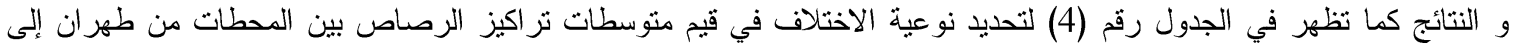

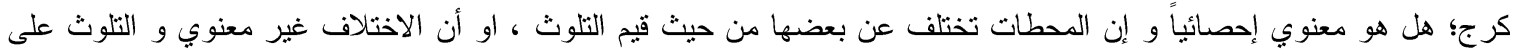
طول الطرق من طهران إلى مدينة كرج هو واحد. بينت النتائج الموضحة في الجدول رقم (4) أنه بين المحطنين الأولى و الر ابعة لايوجد

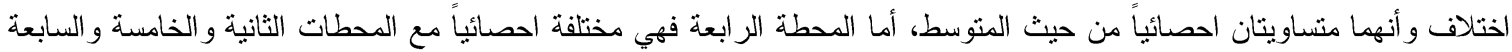

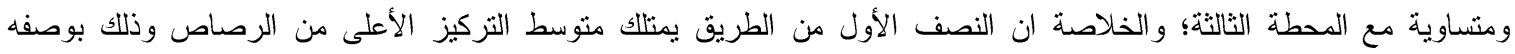

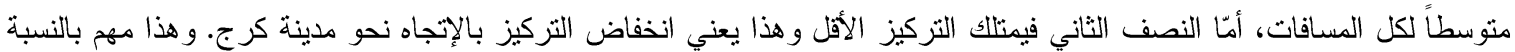

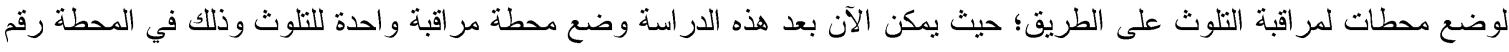
أربعة. 
Journal of University of Babylon for Engineering Sciences, Vol. (26), No. (8): 2018.

\begin{tabular}{|c|c|c|c|c|}
\hline \multicolumn{5}{|c|}{ جدول رقم (4): الفروق المعنوية بين متوسطات تركيز الرصاص المسجلة في محطات الدراسة } \\
\hline Sig $=P$ & 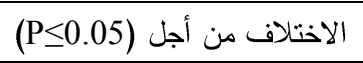 & رمز المحطة & رقم المحطة & نرتيب الاختلافات المعنوية بناء على نتائج التحليل \\
\hline 0.000 & معنوي & $E-F$ & $5-4$ & 1 \\
\hline 0.000 & معنوي & $\mathrm{C}-\mathrm{E}$ & $4-2$ & 2 \\
\hline 0.008 & 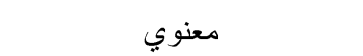 & $\mathrm{D}-\mathrm{F}$ & $3-5$ & 3 \\
\hline 0.008 & 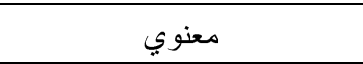 & $\mathrm{C}-\mathrm{D}$ & $2-3$ & 4 \\
\hline 0.035 & معنوي & $\mathrm{E}-\mathrm{H}$ & $4-7$ & 5 \\
\hline $0.05<$ & غير معنوي & - & بقىة & 6 \\
\hline
\end{tabular}

أمّا بالنسبة للتغيرات في متوسط تركيز الرصاص بالعلاقة مع تغير المسافة عن حافة الطريق، الجدول رقم (5) يشرح

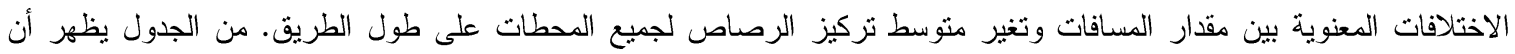

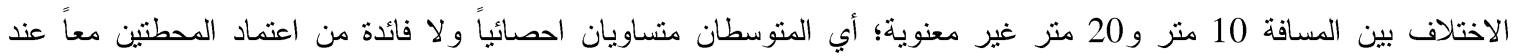

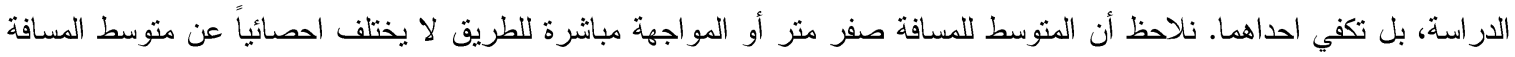

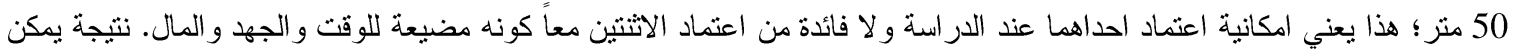
العتماد على المسافات (0، 10، 100) متر عند مر اقبة التلوث على الطريق لمسافات مختلفة.

\begin{tabular}{|c|c|c|c|}
\hline \multicolumn{4}{|c|}{ جدول رقم (5): الفروق المعنوية بين تراكيز الرصاص المسجلة ضمن مسافات مختلفة عن حافة الطريق } \\
\hline Sig $=\mathrm{P}$ & الاختلاف من أجل (P<0.05) & مقدار المسافة بالمتر & ترتيب الاختلافات المعنوية بناء على نتائج التحليل \\
\hline 0.000 & معنوي & $0-100$ & 1 \\
\hline 0.001 & معنوي & $50-100$ & 2 \\
\hline 0.001 & معنوي & $0-10$ & 3 \\
\hline $0.05<$ & غير معنوي & بقىه & 4 \\
\hline
\end{tabular}

4.4.تحليل الإرتباط بين تركيز الرصاص للمحطات المختلفة ويُعد المسافة عن الطريق

تم اعتماد تحليل معامل الارتباط 11 كون الييانات لا تتبع للتوزيع الطبيعي. بيّنت النتائج عدم وجود

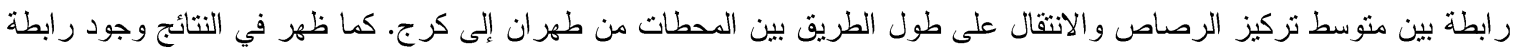

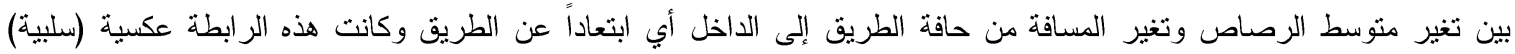
ومعنوية ومقدار ها (0,400) (-0,400 *** ، P=0.004)، أي بالانتقال بعيداً عن الطريق بقل متوسط تركيز الرصاص برابر ابطة خطية بين مقدار المسافة ومقدار المنتوسط.

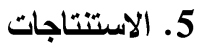

إن الطريقة التحليلية لتقييم الأثر البيئي لطريق طهران- كرج عن طريق أخذ عبنات من التربة الموجودة على أطراف

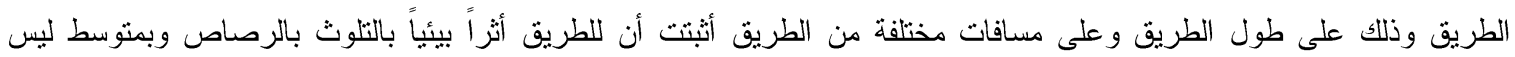

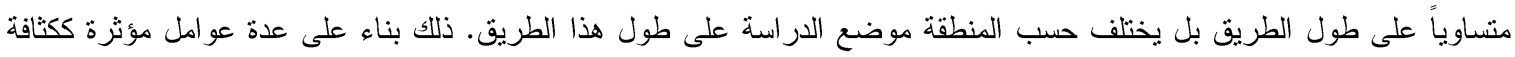

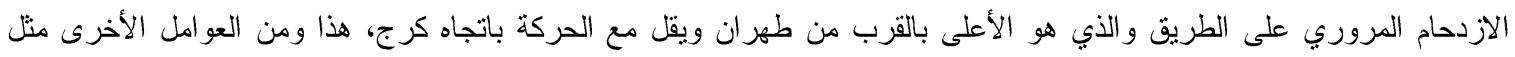

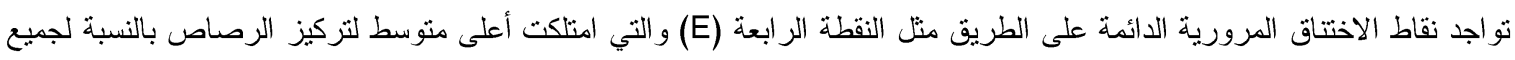

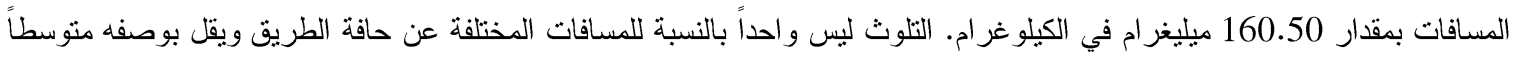

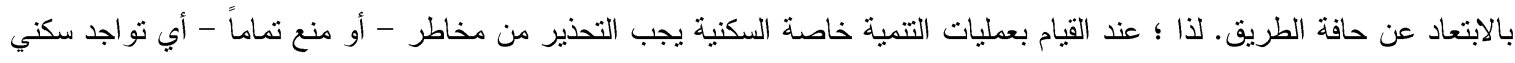


بالقرب من الطريق و اختصاص المناطق المحيطة بالطريق حتى مسافة 100 منز لأعمال توسعة الطريق و المنشآت الخدمية الخاصة

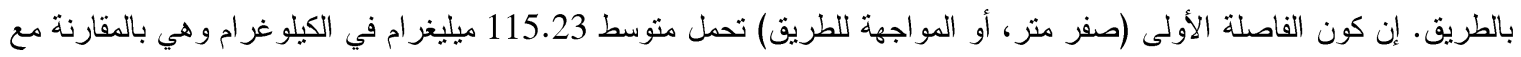
معظم المتوسطات المسجلة للرصاص في الجدول رقم (4)، ويحسب من الطرق الأعلى تلوثاً بالنسبة لبقية دول العالم. صحيح ان متوسط تركيز الرصاص على طول الطريق و على مسافات مختلفة لم يتجاوز الحد المسموح به في الثقارير العالمية المذكورة في الجدول رقم (3)؛ إلّا أنه يجب التبّّه إلى كون بعض النقاط المسجلة على الطريق تحمل متوسطات مرتفعة ومنها ما تجاوز الحد المسموح بثيء طفيف ولكن كإنذار للمستقبل كالنقة الموجودة في المحطة الأولى (B) بالقرب من طهران و الفاصلة صفر، حيث امتلكت متوسط نركيز رصاص (302 ميليغرام في الكيلو غرام). هذا إضافة لنقاط أخرى سجلت بمتوسطات قريبة لل 200 ميليغر ام في الكيلوغرام وذلك للمحطات (D) وE. في هذه النقاط خاصة للفاصلة الأولى ينصح بوضع حرم من الأشجار أبرية الأوراق خاصة من الصنوبر كونها ذات سطح نوعي ورقي أكبر بالمقارنة مع الأنواع الأخرى، وتستطيع استقبال ذرات بشكل كيير ومتحملة للملوثات، بالإضافة إلى عملها مصدات للرياح لكسر وتوزيع الرياح المحملة بالملوثات وخاصة الرصاص الآتية من الطريق على مساحات أوسع و إنقاص ثركيزه المتمركز في نقطة معينة إلى العتبات المسموحة للتلوث.

الثكر الجزيل للأساتذة الذين أثرفوا على سير هذا البحث. أشكر أيضاً جميع الباحثين الذي ذكرتهم في المصادر والذين أخذت ملاحظاتي منهم و أخص بالإمتتان و التقدير لجنة التحكيم التي قيمت هذا العمل ورفعت من شأنه وأغنتتي بالملاحظات القيّة.

\section{CONFLICT OF INTERESTS.}

There are no conflicts of interest.

\section{References}

[1] T. Cahill, D. Barnes, J. Lawton, R. Miller, N. Spada, R. Willis and S. Kimbrough, "Transition metals in coarse, fine, very fine and ultra-fine particles from an interstate highway transect near Detroit," Atmospheric Environment, vol. 145, pp. 158-175, 2016.

[2] A. Mohamadi, S. Mohamadi and M. Saiedi, "Environmental impact assessment of construction and using Tehran- shomal road," in International Conference on Environmental Science and Technology (Farsi), Tehran, 2016.

[3] N. Saffaiean, M. Shokri and B. Jabbarian Amiri, "Environmental impact assessment of north iran development by using distruction model," Environmental science (Farsi), vol. 30, pp. 1-8, 2002.

[4] A. Kabata-Pendias, Trace elements in soils and plants, Fourth Edition, Boca Raton United States of America: CRC Press, 2011.

[5] R. Sutherland and C. Tolosa, "Multi-element analysis of road-deposited sediment in an urban drainage basin, Honolulu, Hawaii," Environmental Pollution, vol. 110, no. 3, p. 483-495, December 2000.

[6] J. H. Duffus, "Heavy metals_A Meaningless Term?," pure and applied chmistry, vol. 74, no. 5, p. 793-807, 2002.

[7] A. Thorpe and R. M. Harrison, "Sources and properties of non-exhaust particulate matter from road traffic: A review," Science of The Total Environment, vol. 400, no. 1-3, p. 270-282, August 2008.

[8] M. Werkenthin, B. Kluge and G. Wessolek, "Metals in European roadside soils and soil solution - A review," Environmental Pollution, vol. 189, pp. 98-110, June 2014.

[9] A. Christoforidis and . N. Stamatis, "Heavy metal contamination in street dust and roadside soil along the major national road in Kavala's region, Greece," Geoderma, vol. 151, no. 3-4, p. 257-263, 15 July 2009. 
[10] M. Huber, . A. Welker and B. Helmreich, "Critical review of heavy metal pollution of traffic area runoff: Occurrence, influencing factors, and partitioning," Science of The Total Environment, vol. 541, p. 895-919, 15 January 2016.

[11] Y. Nazzal, M. A. Rosen and A. M. Al-Rawabdeh, "Assessment of metal pollution in urban road dusts from selected highways of the Greater Toronto Area in Canada," Environmental Monitoring and Assessment, vol. 185, no. 2, p. 1847-1858, February 2013.

[12] N. Morse, . M. T. Walter, D. Osmond and W. Hunt, "Roadside soils show low plant available zinc and copper concentrations," Environmental Pollution, vol. 209, p. 30-37, February 2016.

[13] P. Tamuly and A. Devi, "Heavy metal contamination of roadside topsoil in some areas of Golaghat and Jorhat district along national highway-37, Upper Assam, India," INTERNATIONAL JOURNAL OF ENVIRONMENTAL SCIENCES, vol. 5, no. 2, pp. 472-481, 2014.

[14] K. Swaileh, R. Hussein and S. Abu-Elhaj, "Assessment of heavy metal pollution in roadside surface soil and vegetation from the West Bank," Archives of Environmental Contamination and Toxicology, vol. 47, no. 1, pp. 23-30, Jul 2004.

[15] Chmlab Group, Filtration \& microfiltration new possibilities in filtration, Barcelona-spain: Chmlab Group, 2017.

[16] Anonymous, "Reportes 87/EC of 5 December. European Commission Directive," 2005a, European Union, Commission of the European Communitie.

[17] Anonymous, "The Limits of Pollutants in Food," State Environmental Protection Administration of China, Beijing, China, 2005c.

[18] Anonymous, "Environmental Quality Standard for Soils, Chinese Environmental Protection Administration," Environmental Science Press, China, 1995.

[19] D. Renouf, "Proposed National Enviromental Standard for Assessing and Managing Contamnant in Soil. Guldelines for the Safe Application of Biosoilds to Land in New Zealand," NZWWA and Office of the Minister for the Environment, New Zealand, 2010. 